\title{
Optimization of Time-Partitions for Mixed-Criticality Real-Time Distributed Embedded Systems
}

\author{
Domiţian Tămaş-Selicean and Paul Pop \\ DTU Informatics \\ Technical University of Denmark \\ Kongens Lyngby, Denmark \\ Email:dota@imm.dtu.dk and paul.pop@imm.dtu.dk
}

\begin{abstract}
In this paper we are interested in mixed-criticality embedded real-time applications mapped on distributed heterogeneous architectures. The architecture provides both spatial and temporal partitioning, thus enforcing enough separation for the critical applications. With temporal partitioning, each application is allowed to run only within predefined time slots, allocated on each processor. The sequence of time slots for all the applications on a processor are grouped within a Major Frame, which is repeated periodically. We assume that the safety-critical applications (on all criticality levels) are scheduled using static-cyclic scheduling and the noncritical applications are scheduled using fixed-priority preemptive scheduling. We consider that each application runs in a separate partition, and each partition is allocated several time slots on the processors where the application is mapped. We are interested to determine the sequence and size of the time slots within the Major Frame on each processor such that both the safety-critical and non-critical applications are schedulable. We have proposed a Simulated Annealing-based approach to solve this optimization problem. The proposed algorithm has been evaluated using several synthetic and real-life benchmarks.
\end{abstract}

Keywords-mixed-criticality; real-time systems; temporalpartitioning

\section{INTRODUCTION}

Depending on the particular application, an embedded system has certain requirements on performance, cost, dependability, size etc. In a hard real-time embedded system the "correctness of the system behavior depends not only on the logical results of the computations, but also on the physical instant at which these results are produced" [1]. Safety is a property of a system that will not endanger human life or the environment. Safety-Integrity Levels (SILs) capture the required protection against failure when building a safety-critical embedded system, and will dictate the development processes and certification procedures that have to be followed. There are four SIL levels, ranging from 4 (most critical) to 1 (least critical).

Many such applications, following physical, modularity or safety constraints, are implemented using distributed architectures, composed of several different types of hardware components (called nodes), interconnected in a network. The application software running on such distributed architectures is composed of several functions. The way the functions have been distributed on the architecture has evolved over time. Initially, in automotive and aerospace applications, for example, each function was running on a dedicated hardware node, allowing the system integrators to purchase nodes implementing required functions from different vendors, and to integrate them together into their system (this approach is also called a "federated architecture"). However, the number of such nodes in the architecture has exploded, reaching over one hundred in an airplane or a high-end car, leading to increased wiring, increased costs, size, weight and power consumption.

These trends have created a huge pressure to reduce the number of nodes, use the resources available more efficiently, and thus reduce costs. This is achieved through the integration of several functions in one node (also called an "integrated architecture"). The same trends are driving the integration of several levels of safety-criticality onto the same node, together with non safety-critical functions. The "Research Agenda for Mixed-Criticality Systems" [2] defines a mixed-criticality system as "an integrated suite of hardware, operating system and middleware services and application software that supports the execution of safetycritical, mission-critical, and non-critical software within a single, secure computing platform". The current practice to mixed-criticality systems is to physically separate the different criticality functions in different hardware components, so they cannot influence each other.

In avionics, the proposed integration solution is based on "Integrated Modular Avionics" (IMA) [3], which allows the integration of mixed-criticality functions onto the same node as long as there is enough spatial and temporal partitioning [3]. A similar problem is faced in many other industries. This is coupled with the trend towards using multi-core systems, where several processors can be integrated onto a single chip, decreasing the costs, power consumption, size, and increasing the performance through parallelization [4]. In Europe, multi-cores are addressed through EU projects such as RECOMP ("Reduced certification cost for trusted multi-core platforms" [4]), which has the goal to "define a European standard reference technology for mixed-criticality multi-core systems supported by the European tool vendors". 
In this paper we consider heterogeneous distributed embedded systems that have to implement hard real-time applications with different Safety-Integrity Levels (SILs), including non-critical functions. We assume that the hardware and software architecture provides both spatial and temporal partitioning, thus enforcing enough separation for the mixed-criticality applications. The system architecture is presented in Section III, which includes a discussion on partitioning. Such partitioned architectures are available not only in avionics (where IMA is used), but will also be available in other application areas [4]. We consider that the mapping of tasks to the processors is given.

There are two basic approaches for handling tasks in realtime applications [1]. In the event-triggered approach (ET), activities are initiated whenever a particular event is noted. In the time-triggered (TT) approach, activities are initiated at predetermined points in time. There has been a long debate in the real-time and embedded systems communities concerning the advantages of TT and ET approaches [5], [1]. Safety-critical applications are typically designed using a TT approach to ensure both the predictability of worst-case behavior, and high resource utilization [1]. One disadvantage of the TT approaches is their lack of flexibility, which is an important aspect in non-critical systems.

Therefore, in this paper, we assume that the safetycritical (SC) applications are scheduled using static-cyclic scheduling (SCS) and the non-critical (NC) applications are scheduled using a fixed-priority preemptive scheduling (FPS) policy. For the bus we use the Universal Communication Model [6], which can model both TT and ET traffic. Section II presents the application model. Note that our model, based on [7], is more general and allows any assignment of scheduling policies to applications (i.e., FPS for SC applications), including a combination of TT and ET tasks within the same application. Moreover, although we assume that NC applications are hard real-time, soft realtime functions can also be integrated with fixed-priority preemptive scheduling using a technique such as the Constant Bandwidth Server (CBS) [8], where the server is seen as a hard task providing a desired level of service to soft tasks, issue discussed by us in [9].

Safety-critical real-time applications have to function correctly and meet their timing constraints even in the presence of faults. Fault tolerance can be addressed with hardware architecture solutions, such as TTA [1], or software-based solutions such as re-execution, replication and checkpointing. In [10] we have shown how checkpointing and active replication can be combined in an optimized implementation that leads to a schedulable fault-tolerant application without increasing the amount of employed resources. Hence, in this paper we do not address the issue of fault-tolerance (which is orthogonal to our problem), and we assume that the designer has developed the SC applications such that they provide the required level of fault-tolerance.

\section{A. Contribution}

In this paper we consider that each application runs in a separate partition, and we are interested to determine the sequence and length of the time partitions on each processor such that both the SC and NC applications are schedulable. The exact problem formulation is presented in Section IV and illustrated in Fig. 1. This is the first time, to our knowledge, that such a problem has been addressed.

We have proposed a Simulated Annealing-based approach (SA) to solve this optimization problem, which is presented in Section V, and evaluated using several benchmarks in Section VI. SA decides the sequence (order) of partitions and their length, for each processor.

For each tentative solution produced by SA (consisting of the sequence and length of partition slices) we determine, as follows, if the deadlines are satisfied and how much slack is available (for future upgrades, for example). We first run a List Scheduling-based algorithm (Section V-B) for the SCS applications, to obtain the schedule tables within the defined partitions, and then a Response-Time Analysis (Section V-C) for the FPS applications, which we have extended to obtain the worst-case response times considering the interruption from other time-partitions.

This our is first attempt to solve the problem of optimizing the time-partitions, hence we have decided to use SA because of its simplicity, although, in our experience, a Tabu Search meta-heuristic may produce better results. The right choice of optimization approach depends on the particular problem, and we plan to investigate which is the best approach in our future work. Note that an approach based on Integer Linear Programming can also be used to determine the partitions, but it may be difficult to integrate with the schedulability analysis.

\section{B. Related Work}

Lee et al. [11] consider an IMA-based system where all tasks are scheduled using FPS. The time-partition optimization problem is formulated as a static cyclic scheduling problem, where the partitions are statically scheduled such that the FPS tasks are schedulable. A similar approach to IMA is used in the DEOS operating system [12], with the difference that FPS is used for scheduling both the partitions (which are normally scheduled using SCS) and the tasks. Binns [12] has proposed several slack-stealing approaches, where the unused time in one partition is given to the other partitions, thus the partitions are implicitly adjusted online.

The problem of the optimization of time-partitions has been addressed at the bus level, but without considering partitions at the processor level, mixed-criticality applications and different scheduling policies. Researchers have shown how a Time-Division Multiple Access bus such as the TTP [13] and a mixed TT/ET bus such as FlexRay [14] can be optimized to decrease the end-to-end delays. 
In the context of mixed TT/ET non-critical systems, Pop et al. [7] have shown how the static schedules can be optimized such that both the TT applications (scheduled using SCS) and the ET applications (scheduled using FPS) are schedulable.

There are several works where mixed-criticality tasks are addressed, but researchers assume that tasks of different criticality share the same processor with little or no separation (i.e., there is no spatial-partitioning). Baruah et al. [15] propose a task model that can capture mixedcriticality functions, together with an associated schedulability analysis. Niz et al. [16] discuss the issue of "criticality inversion", similar to the classical priority inversion problem, and propose a "zero-slack scheduling" scheme for such a context.

We have addressed the optimization of CBS-server capacity in the context of mixed hard and soft real-time tasks [9], such that the hard tasks are schedulable and the quality of service for the soft tasks is maximized. CBS-servers provide a time-partitioning between hard and soft tasks.

\section{Application Model}

The set of all applications in the system is denoted with $\Gamma$. A function $F: \Gamma \rightarrow\{S C, N C\}$ captures if an application $\mathcal{A}_{i} \in \Gamma$ is safety-critical (SC) or non-critical (NC). We do not distinguish among different criticality levels for the safetycritical applications. We assume that each SC application has been developed according to the certification requirements for the particular SIL. We model an application as a directed, acyclic graph $\mathcal{G}(\mathcal{V}, \mathcal{E})$. The graph is polar, which means that there is a source node, which is a node that has no predecessors and a sink node that has no successors. Each node $\tau_{i} \in \mathcal{V}$ represents one task.

The tasks are mapped on a distributed heterogeneous architecture, described in the next section. The mapping of each task $\tau_{i}$ is denoted by the function $M: \mathcal{A} \rightarrow \mathcal{N}$, where $\mathcal{N}$ is the set of processing elements (PEs) in the architecture. Each task $\tau_{i}$ is characterized by a worst-case execution time $C_{i}$ (on the PE that is assigned to for execution).

Communication between tasks mapped to different PEs is performed by message passing over the bus. We assume that the message sizes $s_{m_{i}}$ of each message $m_{i}$ are known. An edge $e_{i j} \in \mathcal{E}$ from $\tau_{i}$ to $\tau_{j}$ indicates a synchronous communication: $\tau_{j}$ waits for the output of $\tau_{i}$. Tasks can also communicate asynchronously through buffers, i.e., a reader task will block if the buffer is empty and a writer task will block if the buffer is full. In this case, we assume that the buffer sizes have been determined such that there is no overflow or underflow. Such a communication is not captured explicitly in our model.

For SCS applications, a deadline $D_{\mathcal{G}_{i}} \leq T_{\mathcal{G}_{i}}$, where $T_{\mathcal{G}_{i}}$ is the period of $\mathcal{G}_{i}$, is imposed on each task graph $\mathcal{G}_{i}$. If dependent tasks are of different periods, they are combined into a merged graph capturing all activations for the hyperperiod (LCM of all periods). Release times of some tasks as well as multiple deadlines can be easily modeled by inserting dummy nodes between certain tasks and the source or the sink node respectively. These dummy nodes represent tasks with a certain execution time but which are not allocated to any PE. Thus, by meeting the global deadline, all the local deadlines and release times are guaranteed [17].

Regarding FPS tasks, we use the model from [7], which considers arbitrary deadlines and release times, and also takes into account dependencies. The tasks' priorities are specified by the designer. Thus, for each task $\tau_{i}$ we assume that we know its period $T_{i}$ and deadline $D_{i}$.

\section{SySTEM MODEL}

We consider architectures composed of a set $\mathcal{N}$ of PEs which share a broadcast communication channel. Every PE $N_{i} \in \mathcal{N}$ consists, among others, of a communication controller and a CPU. We assume that the applications are separated using a temporal- and space-partitioning scheme similar to IMA. Space partitioning uses mechanisms such as a Memory Management Unit (MMU) to ensure that, for example, applications running on different partitions cannot corrupt the memory for the other applications. A detailed discussion about space-partitioning is available in [3].

Each application $\mathcal{A}_{j}$ is allowed to execute only within its defined partition $P_{j}$. Although each SC application is running on a different partition to ensure separation, we assume that tasks from the NC applications can share the same partition. On a processing element $N_{i}$, a partition $P_{j}$ is defined as the sequence $P_{i j}$ of $k$ partition slices $p_{i j}^{k}, k \geq 1$. A partition slice $p_{i j}^{k}$ is a predetermined time interval in which the tasks of application $\mathcal{A}_{j}$ mapped to $N_{i}$ are allowed to use the PE. All the slices on a processor are grouped within a Major Frame (MF), that is repeated periodically. The period $T_{M F}$ of the major frame is given by the designer and is the same on each node. Several MFs are combined together in a system cycle that is repeated periodically, with a period $T_{\text {cycle }}$.

In Fig. $1 \mathrm{~d}$ we have 2 PEs, $N_{1}$ and $N_{2}$, and 3 applications, $\mathcal{A}_{1}, \mathcal{A}_{2}$ and $\mathcal{A}_{3}$. Application $\mathcal{A}_{2}$ has 3 tasks, $\tau_{5}, \tau_{6}$ and $\tau_{7}$. Task $\tau_{6}$ executes in partition slices $p_{12}^{1}$ and $p_{12}^{2}$ allocated on $N_{1}$. When a task does not complete during the allocated slice, as it is the case with $\tau_{6}$, its execution is suspended. The time overhead due to partition switching is denoted with $t_{O}$. Our approach takes into account the partition switching overheads. Tasks $\tau_{5}$ and $\tau_{7}$ execute in partition slices $p_{22}^{1}$ and $p_{22}^{2}$, respectively, allocated on $N_{2}$. The sequence and length of the partition slices in a MF are the same (on a given PE), but the contents of the slices can differ. In the example in Fig. 1d all application tasks execute within one MF. However, an application can extend its execution over several MFs, as long as the deadlines are satisfied.

Each partition can use its own scheduling policy. We assume that SC applications are scheduled using non- 
preemptive static-cyclic scheduling, whereas NC applications are scheduled using preemptive fixed-priority scheduling. The start time of each SC task $\tau_{i}$ in the schedule table is denoted by $t_{i}$. The NC tasks have unique priorities denoted by prio $_{i}$.

We consider that processing elements are interconnected using a broadcast bus. We assume that the communication protocol has mechanisms to enforce partitioning at the bus level. For example, space partitioning is attained in SAFEBus [18] by mapping the messages to unique locations in the inter-module memory, protected by a memorymapping hardware in the host, while temporal partitioning is achieved in TTP [1] by enforcing a Time-Division Multiple Access scheme.

We have shown how realistic bus protocols such as TTP [17] and FlexRay (that combines TT and ET traffic) [14] can be taken into account during the design. However, in this paper we assume a more general bus access scheme, called Universal Communication Model (UCM) [6], where the communication cycle is partitioned into static and dynamic phases, see [7] for more details. The SC applications will transmit messages in the static phases, whereas the NC applications use the dynamic phases.

\section{Problem Formulation}

The problem we are addressing in this paper can be formulated as follows: given (1) a set $\Gamma$ of applications of mixed criticality levels, (2) an architecture consisting of a set $\mathcal{N}$ of processing elements, (3) the size of the major frame $T_{M F}$ and (4) the application cycle $T_{c y c l e}$, we are interested to find an implementation $\Psi$ such that all applications meet their deadlines. Deriving an implementation $\Psi$ means deciding on (1) the set $\mathcal{P}$ of partition slices on each processor and (2) the set of schedules $\mathcal{S}$ for the SC applications.

Let us illustrate the problem using the example in Fig. 1 where we have two SC applications, $\mathcal{A}_{1}$ and $\mathcal{A}_{2}$, and one NC application, $\mathcal{A}_{3}$ (see Fig. 1e). For the SC applications, each task has next to it the PE it is mapped to and the worst-case execution time. The period and deadline for the applications are presented under the application graph. The NC tasks are scheduled using FPS and thus have their worst-case execution time $C_{i}$, deadline $D_{i}$, period $T_{i}$, priority and their PE, specified in the table. We have set $T_{M F}=T_{c y c l e}=120 \mathrm{~ms}$. To simplify the discussion, we assume that all NC tasks are released at time 0 , that there is no partition switch overhead and the communication costs are ignored.

Note that, we consider the mapping of tasks to processing elements as fixed, and given as indicated in the figure. Very often, the mapping decision is taken based on the experience and preferences of the designer, considering aspects such as the functionality implemented by the task and the type of processing elements available, legacy constraints, proximity to sensors and actuators. This could be the reason, for example, that tasks $\tau_{1}$ and $\tau_{2}$ of $\mathcal{A}_{1}$ are mapped to different (a)

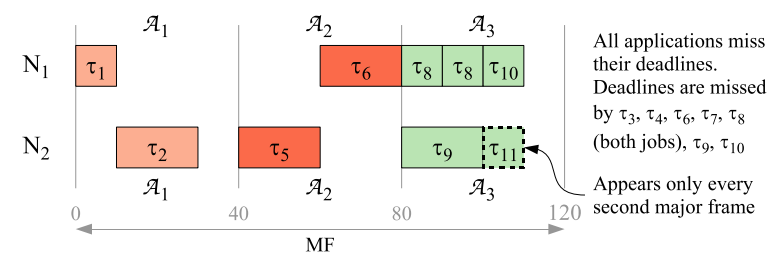

(b)

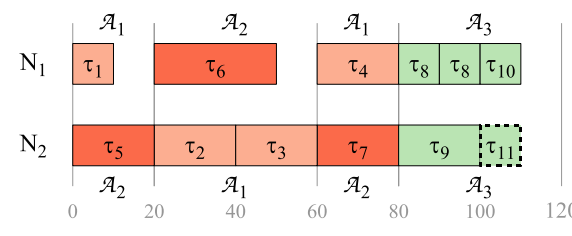

SC applications mee their deadlines. NC application miss theirs. Deadlines are missed by: $\tau_{8}$ and $\tau_{9}$

(c)

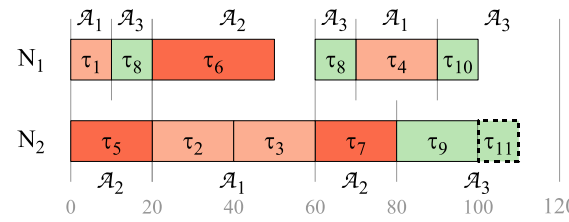

Deadlines are missed only by the $\mathrm{NC}$ task $\tau_{9}$ on $\mathrm{N}_{2}$. All other tasks meet their deadlines

(d)

\begin{tabular}{|c|c|c|c|c|c|c|}
\hline \multirow[b]{2}{*}{$\mathrm{N}_{1}$} & $\mathfrak{A}_{1}, \mathcal{A}_{3}$ & & $\mathcal{A}_{2}$ & $\mathcal{A}_{3}, \mathcal{A}_{2}$ & $\mathcal{A}_{1}$ & \\
\hline & \begin{tabular}{|l|l|l}
$\tau_{1}$ & $\tau_{8}$ & $\tau_{1}$ \\
\end{tabular} & 10 & $\tau_{6}$ & \begin{tabular}{|l|l|}
$\tau_{8}$ & $\tau_{6}$ \\
\end{tabular} & $\tau_{4}$ & \\
\hline \multirow{3}{*}{$\mathrm{N}_{2}$} & & $\mathrm{p}_{22}^{1}$ & $\mathrm{p}^{1}{ }_{12}$ & $\mathrm{p}^{2}{ }_{12}$ & $\mathrm{p}_{22}^{2}$ & \\
\hline & $\tau_{9}$ & $\tau_{5}$ & $\tau_{2}$ & $\tau_{3}$ & $\tau_{7}$ & $\tau_{11}$ \\
\hline & \begin{tabular}{l|l}
$\mathcal{A}_{3}$ & \\
&
\end{tabular} & $\mathcal{A}_{2}$ & & $\begin{array}{l}t_{1} \\
0\end{array}$ & & ${ }_{100}$ \\
\hline
\end{tabular}

Deadlines are met for the all the applications

(e)

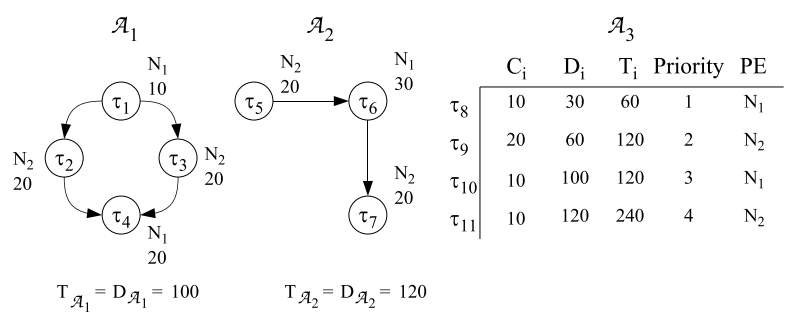

Figure 1: Motivational example

processing elements. Many tasks, however, do not exhibit certain particular features or requirements which lead to an obvious mapping decision. We plan to address the problem of mapping optimization in our future research.

A simple way to do the partitioning is to divide the major frame equally among the 3 applications and to use the same partitioning slices on each PE, as depicted in Fig. 1a. The thin light grey lines are the borders for the partitions slices. Above, respectively under, each partition slice is specified the application it is assigned to. In this case, none of the applications meet their deadlines. Tasks $\tau_{3}$ and $\tau_{4}$ from $\mathcal{A}_{1}$ and $\tau_{7}$ from $\mathcal{A}_{2}$ do not even fit into the system cycle. Note that the deadlines for the NC tasks are measured from their release time. Task $\tau_{8}$ is released twice during the MF, at time 0 and 60 . Task $\tau_{11}$ is released every second MF. The scheduling of SC tasks and the schedulability analysis for the NC tasks are presented in the next section.

In order to better accommodate the SC applications, we can try to adjust the size of the slices and introduce a new 


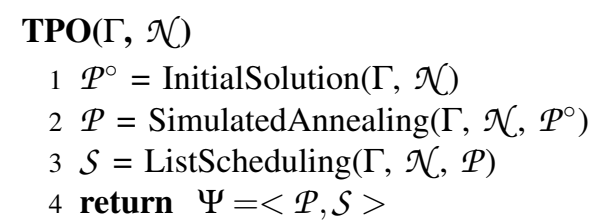

Figure 2: Optimization strategy

slice for $\mathcal{A}_{1}$ on $N_{1}$ and for $\mathcal{A}_{2}$ on $N_{2}$, as shown in Fig. $1 \mathrm{~b}$. The SC applications meet their deadlines, but the NC tasks $\tau_{8}$ and $\tau_{9}$ miss in this case theirs. Note that although the slices have the same sizes on the two PEs, they are assigned to different applications.

Fig. 1c presents a way to make the NC task $\tau_{8}$ on $N_{1}$ meet its deadline. The extra space from the first partition slice associated to $\mathcal{A}_{1}$ on $N_{1}$ is assigned to $\mathcal{A}_{3}$, and the second partition slice of $\mathcal{A}_{1}$ is shifted to the right. In this case, both jobs of $\tau_{8}$ will meet their deadlines. However the NC task $\tau_{9}$ on $N_{2}$ still has a deadline miss.

With the solution proposed in Fig. 1d the partitioning has been optimized such that all deadlines are met. In addition, we have also created 3 unused partition slices, depicted with a light grey rectangle, which can be used, for example, for future upgrades. This was managed by moving the time partition slice for the $\mathrm{NC}$ task $\tau_{9}$ at the beginning of the partition table on PE $N_{2}$ and by splitting the SC task $\tau_{6}$ on PE $N_{1}$ to execute in two different partition slices.

This example shows that the sequence and length of the partition slices has to be carefully optimized in order to find schedulable implementations.

\section{Optimization OF TIME-PARTITIONS}

The problem presented in the previous section is NPcomplete [19]. Its complexity depends not only on the number of tasks and processors, but also on the number of partition slices on each processor. In order to solve this problem, we will use the optimization strategy TimePartitioning Optimization (TPO) from Fig. 2. TPO takes as input a set of applications $\Gamma$ and the set of processing elements $\mathcal{N}$, and returns the implementation $\Psi$ consisting of the set of partitions slices $\mathcal{P}$ on each processor and the schedules $\mathcal{S}$ for the SC applications. Our strategy has 3 steps:

(1) in the first step, we determine an initial set of partition slices $\mathcal{P}^{\circ}$, line 1 in Fig. 2. $\mathcal{P}^{\circ}$ consists of a simple straight forward partitioning scheme which allocates for each application $\mathcal{A}_{j}$ a total time on PE $N_{i}$ proportional to the utilization of the tasks of $\mathcal{A}_{j}$ mapped to $N_{i}$. The partitions $P_{i j}$ thus allocated have the same length and they are distributed with a period equal to the smallest period of a task from $\mathcal{A}_{j}$ mapped to $N_{i}$.

(2) In the second step, we use a Simulated Annealing meta-heuristic to determine the set of partition slices $\mathcal{P}$ such that the applications are schedulable and the unused partition space (potentially used for future upgrades) is maximized (Section V-A). The alternatives provided by Simulated Annealing are evaluated using RTA (Section V-C) and List Scheduling (Section V-B).

(3) Finally, given the optimized partitions $\mathcal{P}$ obtained in line 2 in Fig. 2, we use a List Scheduling heuristic (presented in Section V-B) to determine the schedule tables for the SC applications.

\section{A. Simulated Annealing}

Simulated Annealing (SA) is an optimization metaheuristic that tries to minimize the cost function in order to find the global optimum by randomly selecting neighboring solutions of the current solution [20]. The algorithm presented in Fig. 3 takes as input the set of application $\Gamma$, the architecture $\mathcal{N}$ and the initial partitioning $\mathcal{P}^{\circ}$, and returns the best solution found $\mathcal{P}^{\text {best }}$, i.e., with the smallest cost function (see line 8 in Fig. 3). In order to escape local minima, worse solutions are sometimes accepted with a probability depending on a control parameter called temperature and on the deterioration of the cost function (see lines 10 to 13 in Fig. 3). Before we call SA we merge all $\mathrm{NC}$ tasks into a single application, since the NC tasks are allowed to share a partition.

The algorithm is inspired by the process of annealing metals, a thermal process in which a metal is heated past its melting point and then carefully cooled down so that the particles arrange themselves with lower internal energy than the initial solution [20]. The cooling rate of the process influences the quality of the result. The cooling schedule of SA is defined by the initial temperature $T I$, the temperature length $T L$, the cooling ratio $\varepsilon$ and the stopping criterion. The temperature length $T L$ and the cooling ratio $\varepsilon$ decide how fast will the temperature drop. We use a time limit as a stopping criterion (line 17).

We have defined our cost function as follows:

$$
\operatorname{CostF}(P)=w_{S C} \times \delta_{S C}+w_{N C} \times \delta_{N C}
$$

where $\delta_{S C}$ is the degree of schedulability for SC applications and $\delta_{N C}$ is the degree of schedulability for NC applications. These are summed together into a single value using the weights $w_{S C}$ and $w_{N C}$. In case an application is not schedulable, its corresponding weight is a very big number, i.e., a "penalty" value. This allows us to explore unfeasible solutions (which correspond to unschedulable applications) in the hope of driving the search towards a feasible region. In case an application $\mathcal{A}_{i}$ is schedulable, we use for the weight a value given by the designer, depending on the importance of the application. For example, in our experiments we have used weights for the SC application which are several times greater than those for the NC applications. The degree of schedulability is calculated as:

$$
\delta_{S C / N C}= \begin{cases}c_{1}=\sum_{i} \max \left(0, R_{i}-D_{i}\right) & \text { if } c_{1}>0 \\ c_{2}=\sum_{i}\left(R_{i}-D_{i}\right) & \text { if } c_{1}=0\end{cases}
$$




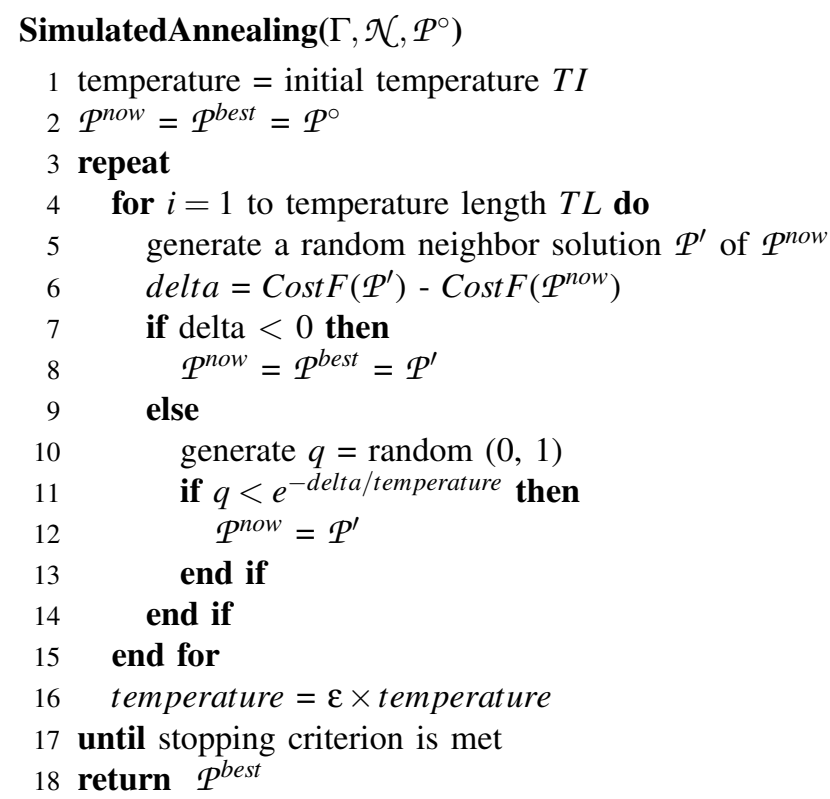

Figure 3: The Simulated Annealing algorithm

For SC applications $\delta_{S C}$ is computed at application level, and thus $R_{i}$ is the response time of the application (i.e., the finishing time of the sink node) as resulted from List Scheduling (see Section V-B), while $D_{i}$ is the deadline of the application. $\delta_{N C}$ is computed at task level. Thus, $R_{i}$ is the worst-case response time and $D_{i}$ is the deadline of each task. The response time for each task is computed according to the response time analysis presented in Section $\mathrm{V}-\mathrm{C}$.

If at least one NC task (or SC application) is not schedulable, there exists one $R_{i}$ greater than the deadline $D_{i}$, and therefore the term $c_{1}$ will be positive. However if all the tasks (applications) are schedulable, this means that each $R_{i}$ is smaller than $D_{i}$, and the term $c_{1}=0$. In this case, we use $c_{2}$ as the degree of schedulability, since it can distinguish between two schedulable solutions.

The neighboring solutions of the current solution $\mathcal{P}^{\text {now }}$ are generated using design transformations (or "moves") applied to $\mathcal{P}^{\text {now }}$. There are 4 types of moves: resize, swap, join and split. The moves are applied to a randomly selected partition slice from a randomly chosen PE.

The resize move, as its name implies, resizes the selected partition slice. This is done either by increasing the size of the partition slice at the expense of a neighboring partition slice, or by decreasing it and giving the extra space to a neighboring slice. The amount with which the partition can be resized is randomly chosen, but we have imposed an upper limit (half the size of the partition). The swap move swaps the chosen partition slice with another randomly chosen partition slice. The join move joins two partition slices belonging to the same application, while the split move splits a partition slice into two, and adds the second

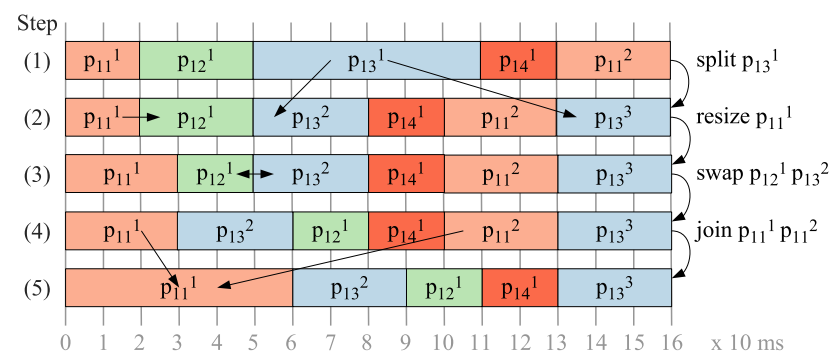

Figure 4: Move examples

slice to the end of the MF. Together with the 4 types of basic moves, we also apply "improved moves". An "improved move" is intended to accelerate the search by performing several basic moves at once.

Fig. 4 depicts the basic moves as they are sequentially performed on a single PE, namely $N_{1}$. As mentioned, the notation $p_{i j}^{k}$ means the $k^{t h}$ partition slice of the application $\mathcal{A}_{j}$ on the processing element $N_{i}$. There are 4 applications, numbered from 1 to 4 , and the first application has 2 partition slices, $p_{11}^{1}$ and $p_{11}^{2}$. The current solution $\mathcal{P}^{\text {now }}$ is presented in Step 1 in Fig. 4. The first move is the split move, which is performed on the partition slice $p_{13}^{1}$ belonging to $\mathcal{A}_{3}$. The slice is split in two equal parts, and the resulting slice is added to the end of the MF. The second move is a resize with $10 \mathrm{~ms}$, which affects $p_{11}^{1}$ at the expense of $p_{12}^{1}$. The third move is a swap of slices $p_{12}^{1}$ and $p_{13}^{2}$. The result is shown in the $4^{\text {th }}$ step. The last move is a join move and as previously mentioned, it can be applied only to partition slices belonging to the same application. For this move we chose the $p_{11}^{1}$ and $p_{11}^{2}$ slices. Once a move has been performed on the partition set $\mathcal{P}^{\text {now }}$, the resulted partition set $\mathcal{P}^{\prime}$ is evaluated using the cost function from (1), which is executed using List Scheduling and Response Time Analysis, presented in the next two sections, respectively.

\section{B. List Scheduling algorithm}

SC applications are scheduled using static-cycling nonpreemptive scheduling. Given a partition set $\mathcal{P}$, we use a List Scheduling (LS)-based heuristic to determine the schedule tables $S$ for each SC application. LS heuristics use a sorted priority list, $L_{\text {ready }}$, containing the tasks ready to be scheduled. A task $\tau_{i}$ is ready if all the predecessor tasks have finished executing and all the incoming messages are received. We use the Modified Partial Critical Path priority function [17] to sort $L_{\text {ready }}$.

We define the response time $R_{i}$ of an application $\mathcal{A}_{i}$ as the time difference between the finishing time of the sink node and the start time of the application. Thus, LS is applied to each SC application. We have modified the classical LS algorithm to take into account the time partitions. Thus, each application is scheduled separately and its tasks are allowed to use only the corresponding partitions slices from $\mathcal{P}$. If 
a partition slice finishes before a SC task has completed its execution (as is the case with $\tau_{6} \in \mathcal{A}_{2}$ in Fig. 1d), we assume that the task is suspended and will continue its execution in the next partition slice allocated to its application. Our LS implementation takes into account the partition switching overhead $t_{O}$. The suspension of the task will take place online, based on the partition scheme $\mathcal{P}$ loaded into the kernel and $t_{O}$ contains the time needed to do a context switch to another partition. LS also schedules the messages on the static segment of the UCM bus.

\section{Response Time Analysis}

$\mathrm{NC}$ applications are scheduled using FPS. To determine the schedulability of NC applications we use a response-time analysis [21] to calculate the worst-case response time $R_{i}$ of every NC task $\tau_{i}$, which is compared to its deadline $D_{i}$. The basic analysis presented in [21] has been extended over the years. For example, the state-of-the-art analysis from [22] considers arbitrary arrival times and deadlines, offsets and synchronous inter-task communication (where a receiving task has to wait for the input of the sender task). Audsley and Wellings [23] have proposed a schedulability analysis for FPS tasks using temporal partitioning (IMA), which, when analyzing a FPS task in a certain partition, considers the other time-partitions as higher priority tasks. This analysis assumes that the deadlines are smaller or equal to the periods, that the tasks are independent, and that the start times of partition slices within a major frame are periodic. Pop et al. [7] have proposed a schedulability analysis for ET tasks, which extends the schedulability analysis with static and dynamic offsets in [22] to consider the influence of the TT tasks on the worst-case response times of the ET tasks.

In this paper, we have extended the analysis from [7] to consider the influence of time-partitions on the schedulability of the NC tasks. In [7], the authors introduce the notion of ET demand and ET availability, used to compute the length of the busy window $w_{i}$, which is needed in order to compute the worst-case response time $R_{i}$ of a task $\tau_{i}$. The busy window $w_{i}$ is the longest time interval during which tasks of priority equal or greater than $\tau_{i}$ are continuously executing [24]. The worst-case response time is determined using (3), considering a certain length of the busy window $w_{i}$, and all the higher priority tasks:

$$
R_{i}=w_{i}-\varphi_{i}-(p-1) \times T_{i}+\phi_{i}
$$

where $p$ is the number of activations of task $\tau_{i}$ in the busy window $w_{i}, T_{i}$ is the period of $\tau_{i}$, the offset $\phi_{i}$ is the earliest activation of $\tau_{i}$ relative to the occurence of the triggering event and the phase $\varphi_{i}$ is the time interval between the critical instant and earliest time of the first activation of $\tau_{i}$. The worst-case response time $R_{i}$ for the task $\tau_{i}$ is the maximum value of the result in (3), considering all the critical instants initiated by higher priority tasks and by $\tau_{i}$ and also all the job instances. During the calculation, if the available time does not satisfy the demand of $\tau_{i}$ then the algorithm increases iteratively the length of the busy window $w_{i}$ which is analyzed [7].

Similar to the notion of ET demand from [7], we introduce $N C$ demand, associated with a NC task $\tau_{i}$ on a time interval $t$, as the maximum amount of CPU time which can be demanded by higher or equal priority NC tasks and by $\tau_{i}$. Fig. 5 shows the analysis for a task $\tau_{i}$, considering the busy window that starts at the critical instant $q T_{i}+t_{c}$, initiated by task $\tau_{a}$ and ends at the moment $q T_{i}+t_{c}+w_{i}$, when all the higher priority tasks $\left(\tau_{a}\right.$ and $\left.\tau_{b}\right)$ and $\tau_{i}$ itself have finished execution, and when all the partition slices interrupting $\tau_{i}$ have finished.

During the busy window $w_{i}$, the demand $H_{i}$ associated with task $\tau_{i}$ scheduled in a partition $P_{k}$ is equal with the length of the busy window which would result when considering that $P_{k}$ would be the only partition on the processor. Thus, similar to [7] and [22], the $N C$ demand is:

$$
\begin{gathered}
H_{i}\left(w_{i}\right)=B_{i}+\left(p-p_{0, i}+1\right) \times C_{i}+ \\
W_{i}\left(\tau_{i}, w_{i}\right)+\sum_{\forall\left(a \in \mathcal{A}_{a} \neq \mathcal{A}_{i}\right)} W_{a}^{*}\left(\tau_{i}, w_{i}\right)
\end{gathered}
$$

where $B_{i}$ is the maximum blocking time for $\tau_{i}$. The job activations of task $\tau_{i}$ during $w_{i}$ are denoted with $p$ and positive values are assigned to instances arriving after $t_{c}$, while zero and negative values indicate the instance arrived before $t_{c}$. Thus, $p_{0, i}$ is the index of the first pending instance of $\tau_{i}$ and is computed as follows:

$$
p_{0, i}=1-n_{i a}=1-\left\lfloor\frac{J_{i}+\varphi_{i}}{T_{i}}\right\rfloor,
$$

where $n_{i a}$ is the number of pending $\tau_{i}$ jobs at $t_{c}$, during the busy window $w_{i}$ initiated by $\tau_{a} . W_{i}\left(\tau_{i}, w_{i}\right)$ is the interference from higher priority tasks $\mathrm{hp}\left(\tau_{i}\right)$ in the same application $\mathcal{A}_{i}$ as $\tau_{i}$ :

$$
W_{i}\left(\tau_{i}, w_{i}\right)=\sum_{j \in h p\left(\tau_{i}\right)}\left(\left\lfloor\frac{J_{j}+\varphi_{i}}{T_{i}}\right\rfloor+\left\lceil\frac{w_{i}-\varphi_{i}}{T_{i}}\right\rceil\right) \times C_{j}
$$

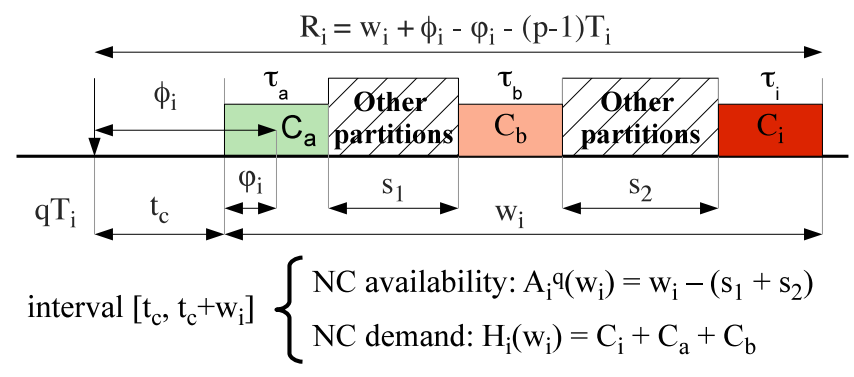

Figure 5: Availability and demand 
and $W_{a}^{*}\left(\tau_{i}, w_{i}\right)$ is the worst-case interference from higher priority $h p_{a}\left(\tau_{i}\right)$ tasks from other applications than the application $\mathcal{A}_{i}$ that $\tau_{i}$ belongs to:

$$
W_{a}^{*}\left(\tau_{i}, w_{i}\right)=\max \left(W_{k}\left(\tau_{i}, w_{i}\right)\right), \forall k \in h p_{a}\left(\tau_{i}\right)
$$

Fig. 5 shows that the $N C$ demand of task $\tau_{i}$ during the busy window $w_{i}$ is the sum of the worst case execution times of the higher priority $\mathrm{NC}$ tasks, $C_{a}$ and $C_{b}$, and the worst-case execution time $C_{i}$ of $\tau_{i}$.

We extend the concept of availability from [7] as the processing time available during $w_{i}$ for $P_{k}$. Considering we are using time partitions and that task $\tau_{i}$ can execute only during its own partition $P_{k}$, the availability is computed by subtracting from $w_{i}$ the time reserved for the "other partitions". In Fig. 5 the other partitions are illustrated with hashed rectangles and their duration denoted with $s_{1}$ and $s_{2}$.

The response time analysis also determines the worst-case response time $R_{m}$ of each message $m$ transmitted on the dynamic segment of the UCM bus. For details, see [7].

\section{EXPERIMENTAL EVALUATION}

For the evaluation of our proposed algorithm we used 10 synthetic benchmarks and 2 real life case studies. The TimePartition Optimization (TPO) algorithm was implemented in Java (JDK 1.6), running on SunFire v440 computers with UltraSPARC IIIi CPUs at $1.062 \mathrm{GHz}$ and $8 \mathrm{~GB}$ of RAM.

In the first set of experiments we were interested to evaluate the proposed TPO strategy in terms of its ability to find schedulable implementations. Thus, we have used 5 synthetic benchmarks with 3 to $5 \mathrm{SC}$ applications (with a total of 15 to $53 \mathrm{SC}$ tasks). All the $\mathrm{NC}$ tasks have been merged into a single NC application, with 5 to 9 tasks. The resulted mixed-criticality system has been mapped on architectures ranging from 2 to 6 processing elements. The mapping has been done such that the utilization on the PEs is balanced and the communication over the bus is minimized. The execution times and message lengths were assigned randomly within the 1 to $19 \mathrm{~ms}$ and 1 to 5 bytes ranges, respectively. The weights used for computing the cost function were $w_{S C}=400$ for SC applications and $w_{N C}=100$ for NC tasks (see Section V-A).

We have used two time limits for the experiments: 10 minutes and 120 minutes. The results obtained with TPO using a time limit of 120 minutes are presented in Table I, under the heading "TPO, 120 min. time limit".

We were interested to determine the quality of our Simulated Annealing-based (SA) TPO strategy. Hence, we have used an exhaustive search to determine the optimal solutions. Since the runtime of the exhaustive search is prohibitively large, we were only able to run it for smaller examples, lines 1, 6 and 7 in Table I. In these cases, our SA-based approach is capable of obtaining (in 120 minutes) solutions which are very close to the optimum. For the benchmarks in lines 1 ,
6 and 7 the difference in term of the cost function is only $4.51 \%, 0.16 \%$ and $1.9 \%$, respectively.

Together with TPO, Table I also presents the results obtained using a Straightforward Solution (SS), which implements the approach from the InitialSolution function presented in Section V. SS is an approach that a good designer would use if TPO would not be available. Columns 2 and 4 in Table I present the number of SC applications, and the NC tasks, respectively. The number of schedulable applications and tasks (out of the total) obtained by our proposed TPO strategy are presented in columns 8 and 9, respectively, while columns 6 and 7 present the results obtained using SS. Columns 10 and 11 represent the percentage increase in the degree of schedulability for SC applications, $\Delta_{S C}$, and NC tasks, $\Delta_{N C}$, (see Section V-A) as obtained by the TPO strategy compared to the SS, considering a time limit of 120 minutes. A negative value for $\Delta_{N C}$ means that our optimization has decreased the degree of schedulability for the $\mathrm{NC}$ tasks in order to guarantee that all SC applications are schedulable. Note that the NC tasks are still schedulable in this case, but their response times have increased, compared to SS, which over-dimensioned the NC partitions. Column 12 represents the average of the percentage increase in the degree of schedulability for the whole system.

We have also run TPO with a time limit of $10 \mathrm{~min}$. TPO is able to obtain schedulable solutions in all cases, except for the case study in line 4 in Table I. The average deviation of the percentage increase of the cost function (as captured by (1)) for the schedulable results, compared to the results obtained with TPO using a 120 min. time limit, is of $10.48 \%$.

As we can see from "Set 1", SS which does not perform optimization, is not able to find schedulable implementations. For example, for the largest benchmark, with 5 SC application and 9 NC tasks mapped on 6 PEs only 3 out of $5 \mathrm{SC}$ applications are schedulable. All the $\mathrm{NC}$ tasks are schedulable. Note that SS leads to schedulable NC implementations. This is because it distributes the partition slices to match the smallest period of the tasks. However, since the slices have equal lengths, there is a lot of wasted space in the schedules of SC applications, which leads to missed SC deadlines. However, by applying our proposed TPO approach, we are able to optimize the time partitions such that all applications are schedulable. We have measured the ability of TPO to improve over SS by using a percentage average increase in the degree of schedulability over all applications, presented in the last column. As we can see there is a dramatic increase in the degree of schedulability over all applications, when using TPO. This means that we can potentially implement the applications on a slower (cheaper) architecture.

In the second set of applications, labeled "Set 2", we were interested to see how TPO performs as the utilization of the system increases. We have mapped 2 to 6 applications on the same architecture of 4 PEs. As we can see, TPO is able 
Table I: Experimental results for benchmarks

\begin{tabular}{|c|c|c|c|c|c|c|c|c|c|c|c|}
\hline \multirow{2}{*}{ Set } & \multicolumn{2}{|c|}{ SC } & \multirow{2}{*}{\begin{tabular}{|l|} 
NC \\
Tasks
\end{tabular}} & \multirow{2}{*}{ PEs } & \multicolumn{2}{|c|}{ SS } & \multicolumn{5}{|c|}{ TPO, $120 \mathrm{~min}$. time limit } \\
\hline & Apps & Tasks & & & $\begin{array}{c}\text { Sched. SC } \\
\text { Apps }\end{array}$ & $\begin{array}{l}\text { Sched. NC } \\
\text { Tasks }\end{array}$ & $\begin{array}{c}\text { Sched. SC } \\
\text { Apps }\end{array}$ & $\begin{array}{l}\text { Sched. NC } \\
\text { Tasks }\end{array}$ & $\Delta_{S C}$ & $\Delta_{N C}$ & $\begin{array}{c}\text { avg. } \% \\
\text { increase in } \delta\end{array}$ \\
\hline \multirow{5}{*}{1} & 3 & 15 & 5 & 2 & 1 of 3 & All & All & All & 1709.76 & $\mid-44.00$ & 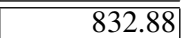 \\
\hline & 3 & 20 & 6 & 3 & 1 of 3 & All & All & All & 107.94 & -53.23 & 27.36 \\
\hline & 4 & 34 & 6 & 4 & None & All & All & All & 169.68 & 7.14 & 88.41 \\
\hline & 4 & 40 & 10 & 5 & None & All & All & All & 147.54 & -0.40 & 73.57 \\
\hline & 5 & 53 & 9 & 6 & 3 of 5 & All & All & All & 542.78 & 14.66 & 278.72 \\
\hline \multirow{5}{*}{2} & $\overline{1} 1$ & 6 & $\overline{6} 6$ & 4 & All & All & $\overline{\text { All }}$ & All & 78.38 & |0.00 & 39.19 \\
\hline & 2 & 12 & 6 & 4 & All & All & All & All & 59.20 & -2.87 & 28.17 \\
\hline & 3 & 20 & 6 & 4 & None & 5 of 6 & All & All & 518.06 & 1453.85 & 985.96 \\
\hline & 4 & 30 & 6 & 4 & 1 of 4 & All & All & All & 211.66 & 0.00 & 105.83 \\
\hline & 5 & 34 & 6 & 4 & 2 of 5 & 5 of 6 & All & All & 466.36 & 673.33 & 569.85 \\
\hline \multirow{2}{*}{3} & $\overline{3}$ & $\overline{19}$ & $\overline{5}$ & $\overline{3}$ & None & 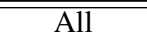 & $\overline{\text { All }}$ & $\overline{\text { All }}$ & 227.33 & |0.57 & 113.95 \\
\hline & 4 & 19 & 6 & 3 & All & All & All & All & 135.29 & -11.56 & 61.87 \\
\hline
\end{tabular}

to find schedulable implementations even as the utilization increases.

Finally, we have also used 2 real life benchmarks derived from the Embedded Systems Synthesis Benchmarks Suite (E3S) version 0.9 [25]. We have used the telecom-mocsyn and auto-indust-cowls benchmarks. In the case of telecommocsyn test case, the applications numbered as 0, 1, 2 and 4 were used as SC, and applications numbered as 3, 5, 6 and 7 were merged into one NC application. In the case of the auto-indust-cowls, the first 3 applications are considered $\mathrm{SC}$, while the last one is NC. In both cases the applications are mapped on an architecture of 3 PEs. The results obtained from these real-life benchmarks confirm the results of the synthetic benchmarks.

\section{CONCLUSiOnS AND Future WORK}

In this paper we have presented a Simulated Annealing based algorithm for the optimization of time-partitions for mixed-criticality real-time distributed embedded systems. The algorithm considers that the applications are separated using a temporal- and spatial-partitioning scheme similar to IMA, that each application runs in a separate time partition, that the SC applications are scheduled using static-cycling scheduling, while the NC applications are scheduled using fixed-priority preemptive scheduling. Two real life examples have been used, as well as 10 synthetic benchmarks to show the effectiveness of the proposed algorithm. We have shown that only by optimizing the sequence and length of the time partitions we are able to obtain schedulable implementations.

The most important extension in our future work will be to consider certification costs. We will extend existing cost models from literature [26] to take into account the additional effort required for certification at a given SafetyIntegrity Level. If two applications of different SILs share a partition, they will have to be certified at the highest SIL level among the two, increasing thus the overall development costs. Our future approach will allow the sharing of parti- tions, which could lead to reduced system costs, and will consider the increased development costs due to increased certification efforts. In this paper we have assumed that the mapping is given, but we plan to include the problem of assigning tasks to processing elements in the overall partitioning optimization problem. Also, in our future work we will investigate which is the best optimization approach, for example using Tabu Search instead of Simulated Annealing.

\section{REFERENCES}

[1] H. Kopetz, Real-Time Systems-Design Principles for Distributed Embedded Applications. Kluwer Academic Publishers, 1997.

[2] J. Barhorst, T. Belote, P. Binns, J. Hoffman, J. Paunicka, P. Sarathy, J. Scoredos, P. Stanfill, D. Stuart, and R. Urzi, "A research agenda for mixed-criticality systems," in CyberPhysical Systems Week, April 2009.

[3] J. Rushby, "Partitioning for avionics architectures: Requirements, mechanisms, and assurance," NASA Langley Research Center, NASA Contractor Report CR-1999-209347, Jun. 1999, also to be issued by the FAA.

[4] R. Ernst, "Certification of trusted mpsoc platforms," 2010, 10th International Forum on Embedded MPSoC and Multicore.

[5] N. Audsley, K. Tindell, and A. Burns, "The end of the line for static cyclic scheduling," in Proc. of Euromicro Workshop on Real-Time Systems, 1993, pp. 36-41.

[6] T. Demmeler and P. Giusto, "A universal communication model for an automotive system integration platform," in Design, Automation and Test in Europe, 2001. Conference and Exhibition 2001. Proceedings. IEEE, 2002, pp. 47-54.

[7] T. Pop, P. Pop, P. Eles, and Z. Peng, "Analysis and optimisation of hierarchically scheduled multiprocessor embedded systems," International Journal of Parallel Programming, vol. 36, no. 1, pp. 37-67, 2008. 
[8] L. Abeni and G. Buttazzo, "Integrating multimedia applications in hard real-time systems," in Proc. of Real-Time Systems Symposium, 1998, pp. 4 -13.

[9] P. K. Saraswat, P. Pop, and J. Madsen, "Task mapping and bandwidth reservation for mixed hard/soft fault-tolerant embedded systems," Real-Time and Embedded Technology and Applications Symposium, IEEE, vol. 0, pp. 89-98, 2010.

[10] P. Pop, V. Izosimov, P. Eles, and Z. Peng, "Design optimization of time- and cost-constrained fault-tolerant embedded systems with checkpointing and replication," Very Large Scale Integration (VLSI) Systems, IEEE Transactions on, vol. 17 no. 3, pp. $389-402$, march 2009.

[11] Y.-H. Lee, D. Kim, M. Younis, J. Zhou, and J. McElroy, "Resource scheduling in dependable integrated modular avionics," in Proc. of Dependable Systems and Networks, 2000, pp. $14-23$.

[12] P. Binns, "A robust high-performance time partitioning algorithm: the digital engine operating system (DEOS) approach," in Conf. on Digital Avionics Systems, vol. 1, 2001, pp. 1B6/1 $-1 \mathrm{~B} 6 / 12$.

[13] P. Pop, P. Eles, and Z. Peng, "Scheduling with optimized communication for time-triggered embedded systems," in Proc. of the Workshop on Hardware/software Codesign, 1999, pp. 178-182.

[14] T. Pop, P. Pop, P. Eles, Z. Peng, and A. Andrei, "Timing analysis of the FlexRay communication protocol," Real-Time Systems, vol. 39, no. 1-3, pp. 205-235, 2008.

[15] S. Baruah, H. Li, and L. Stougie, "Towards the design of certifiable mixed-criticality systems," in Real-Time and Embedded Technology and Applications Symp., 2010, pp. 13 -22 .
[16] D. de Niz, K. Lakshmanan, and R. Rajkumar, "On the scheduling of mixed-criticality real-time task sets," in Proc. of the Real-Time Systems Symposium, 2009, pp. 291-300.

[17] P. Pop, P. Eles, and Z. Peng, Analysis and Synthesis of Communication-Intensive Heterogenous Real-Time Systems. Kluwer Academic Publishers, 2004.

[18] K. Hoyme and K. Driscoll, "SAFEbus," IEEE Aerospace Electronic Systems Magazine, vol. 8, pp. 34-39, 1993.

[19] J. D. Ullman, "NP-complete scheduling problems," J. Comput. Syst. Sci., vol. 10, no. 3, pp. 384-393, 1975.

[20] E. Burke and G. Kendall, Search Methodologies. Springer Science + Business Media, 2005, ch. 7 .

[21] G. C. Buttazzo, Hard Real-Time Computing Systems: Predictable Scheduling Algorithms and Applications. Kluwer Academic Publishers, 1997.

[22] J. Palencia and M. Gonzalez Harbour, "Schedulability analysis for tasks with static and dynamic offsets," in Proc. of Real-Time Systems Symposium, 1998, pp. 26 -37.

[23] N. Audsley and A. Wellings, "Analysing APEX applications," in Real-Time Systems Symp., 1996, pp. $39-44$.

[24] C. Fidge, "Real-time schedulability tests for preemptive multitasking," REAL-TIME SYSTEMS, vol. 14, no. 1, pp. 61-93, JAN 1998.

[25] R. Dick, "Embedded system synthesis benchmarks suite," http://ziyang.eecs.umich.edu/dickrp/e3s/.

[26] J. Axelsson, "Cost models for electronic architecture trade studies," in Proc. of ICECCS, 2000, pp. 229-239. 